\title{
On Generalized Surjective Codes
}

\author{
Jörn Quistorff \\ Department 4 \\ FHTW Berlin (University of Applied Sciences) \\ 10313 Berlin, Germany \\ J.Quistorff@fhtw-berlin.de \\ Jan-Christoph Schlage-Puchta \\ Albert-Ludwigs-Universität \\ Mathematisches Institut \\ Eckerstr. 1 \\ 79104 Freiburg, Germany \\ jcp@math.uni-freiburg.de
}

June 30, 2011

\begin{abstract}
We consider generalized surjective codes, together with their connection to covering codes and covering arrays. We prove new bounds on $\sigma_{q}(n, s ; r)$, the minimal cardinality of a $q$-ary code of length $n$, which is $s$-surjective with radius $r$. For covering codes we deduce the new records $K_{6}(10,7) \leq 18$ and $K_{6}(9,6) \leq 24$.
\end{abstract}

Key words: Covering code, covering array, surjective code

2000 Mathematics Subject Classification: 94B65

\section{Introduction}

Let $Q$ be a $q$-set, e.g. $\mathbb{Z}_{q}=\{0,1, \ldots, q-1\}$, and consider the $n$-dimensional $q$-ary Hamming space, i.e. $Q^{n}$ equipped with the Hamming metric $d_{H}$. We say that a word $y \in Q^{n}$ is $R$-covered by $x \in Q^{n}$ if $d_{H}(x, y) \leq R$.

Definition 1. A word $y \in Q^{n}$ is said to be $R$-covered by a q-ary code $C \subset Q^{n}$ of length $n$ if there is a codeword $c \in C$ that $R$-covers $y$. The code $C$ is called 
$R$-covering if any word $y \in Q^{n}$ is $R$-covered by $C$. Let $K_{q}(n, R)$ denote the minimal cardinality of a q-ary $R$-covering code of length $n$.

For a monograph on covering codes see [6]. An updated table of bounds on $K_{q}(n, R)$ is published online by Kéri [14].

Definition 2. A covering array $C A_{\lambda}(N ; t, k, v)$ is an $N \times k$ array with entries from $\mathbb{Z}_{v}$ such that in every $N \times t$ subarray, each $t$-tuple of $\mathbb{Z}_{v}^{t}$ occurs at least $\lambda$ times. Let $C A N(t, k, v)$ denote the minimum possible number of rows $N$ of a $C A_{1}(N ; t, k, v)$.

Covering arrays with $\lambda=1$ are also known as surjective codes, see [4] and its references as well as [15]. An updated table of upper bounds on $C A N(t, k, v)$ is published online by Colbourn [5]. If in a $C A_{1}(N ; t, k, v)$ each $t$-tuple occurs exactly once then it is called an orthogonal array of index 1 , see [9], and the corresponding code is called a $v$-ary $(k, t)$ MDS code. A common generalization of $R$-covering codes and covering arrays with $\lambda=1$ is the following notion of a generalized surjective code.

Definition 3 (Kéri, Östergård [15]). A q-ary code $C \subset Q^{n}$ of length $n$ is called $s$-surjective with radius $r$ if for any $s$-tuple $\left(k_{1}, \ldots, k_{s}\right) \in \mathbb{Z}_{n}^{s}$ of pairwise distinct coordinates and any s-tuple $\left(x_{1}, \ldots, x_{s}\right) \in Q^{s}$ there is a codeword $c=\left(c_{0}, \ldots, c_{n-1}\right) \in C$ such that $\left|\left\{i \in\{1, \ldots, s\} \mid c_{k_{i}}=x_{i}\right\}\right| \geq s-r$. Let $\sigma_{q}(n, s ; r)$ denote the minimal cardinality of a q-ary code of length $n$, which is s-surjective with radius $r$.

Clearly, $\sigma_{q}(n, n ; r)=K_{q}(n, r)$ and $\sigma_{q}(n, s ; 0)=C A N(s, n, q)$. The generalized surjective codes turned out to be a valuable tool in the theory of covering codes, see $[15,17,7]$.

Whenever we want to prove that a code $C \subset Q^{n}$ is $s$-surjective with radius $r$ we will consider arbitrary $s$ coordinates $k_{1}<\cdots<k_{s}$ from $\mathbb{Z}_{n}$ and an arbitrary $x=\left(x_{1}, \ldots, x_{s}\right) \in Q^{s}$. Then we shall show that there is a codeword $c=\left(c_{0}, \ldots, c_{n-1}\right) \in C$ such that $x$ is $r$-covered by $c^{*}:=$ $\left(c_{k_{1}}, \ldots, c_{k_{s}}\right)$. Sometimes, it will be convenient to label the elements of $\mathbb{Z}_{n} \backslash$ $\left\{k_{1}, \ldots, k_{s}\right\}$ by $l_{1}, \ldots, l_{n-s}$.

This paper is organized as follows. Section 2 discusses recursive bounds. In Section 3, 4 and 5, exact values, lower and upper bounds are presented, respectively. We collect the results for small parameters in the tables of Section 6. 
We would like to thank the referee for greatly improving this paper, in particular for making us aware of several possible improvements in these tables.

\section{Recursive Bounds}

The following recursive bounds are well-known and quite powerful in some situations:

$$
\begin{aligned}
K_{q}\left(n_{1}+n_{2}, R_{1}+R_{2}+1\right) & \geq \min \left\{K_{q}\left(n_{1}, R_{1}\right), K_{q}\left(n_{2}, R_{2}\right)\right\} \\
K_{q_{1} \cdot q_{2}}(n, R) & \leq \sigma_{q_{1}}(n, n-R ; 0) \cdot K_{q_{2}}(n, R) \\
K_{q}(n+s, R+r+1) & \geq \min \left\{\sigma_{q}(n+s, s ; r), K_{q}(n, R)\right\} \\
\sigma_{q}(n+1, s ; r) & \geq \sigma_{q}(n, s ; r) \\
\sigma_{q_{1}+q_{2}}\left(n, s_{1}+s_{2}-1 ; s_{1}+s_{2}-1-r\right) & \leq \sigma_{q_{1}}\left(n, s_{1}, s_{1}-r\right)+\sigma_{q_{2}}\left(n, s_{2} ; s_{2}-r\right) \\
\sigma_{q}(n, s ; R+s-n) & \geq K_{q+1}(n, R)-1 \text { if } R+1 \geq s \\
\sigma_{q+1}(n+1, s+1 ; r+1) & \geq \min \left\{2(q+1), \sigma_{q}(n, s ; r)+1\right\} \text { if } r<s
\end{aligned}
$$

Inequality (1) is from [1], (2) is from [6], (3) and (4) and (5) are from [15], (6) and (7) are from [7].

\section{Theorem 4.}

$$
\sigma_{q}(n+1, s+1 ; r+1) \leq \sigma_{q}(n, s ; r) \leq \sigma_{q}(n, s+1 ; r) .
$$

Proof. Let $C \subset \mathbb{Z}_{q}^{n}$ be a code of cardinality $\sigma_{q}(n, s ; r)$ which is $s$-surjective with radius $r$. Obtain $\bar{C}$ from $C$ by repeating the last component. We shall show that $\bar{C}$ is $(s+1)$-surjective with radius $r+1$. Since $k_{1}, \ldots, k_{s} \in \mathbb{Z}_{n}$ there is a $c=\left(c_{0}, \ldots, c_{n-1}\right) \in C$ such that $\left(x_{1}, \ldots, x_{s}\right) \in \mathbb{Z}_{q}^{s}$ is $r$-covered by $c^{*}$. Set $\bar{c}=\left(c_{0}, \ldots, c_{n-1}, c_{n-1}\right) \in \bar{C}$ then $x$ is $(r+1)$-covered by $\bar{c}^{*}$. This proves the first inequality. The second one is trivial.

The bounds (4) and (8) imply

$$
\sigma_{q}(n, s+1 ; r+1) \leq \sigma_{q}(n, s ; r)
$$

and

$$
\sigma_{q}(n, s ; r) \geq K_{q}(s, r) .
$$

A common generalization of (1) and (3) is the following new result. 


\section{Theorem 5.}

$$
\begin{aligned}
& \sigma_{q}\left(n_{1}+n_{2}, s_{1}+s_{2} ; r_{1}+r_{2}+1\right) \\
& \quad \geq \min \left\{\sigma_{q}\left(n_{1}+n_{2}, s_{1} ; r_{1}\right), \sigma_{q}\left(n_{1}+n_{2}-s_{1}, s_{2} ; r_{2}\right)\right\} \\
& \quad \geq \min \left\{\sigma_{q}\left(n_{1}, s_{1}, r_{1}\right), \sigma_{q}\left(n_{2}, s_{2} ; r_{2}\right)\right\}
\end{aligned}
$$

Proof. The first inequality can be shown analogously to the proof of $[15$, Theorem 2]: Set $M_{1}=\sigma_{q}\left(n_{1}+n_{2}, s_{1} ; r_{1}\right)$ and $M_{2}=\sigma_{q}\left(n_{1}+n_{2}-s_{1}, s_{2} ; r_{2}\right)$. Let $C \subset \mathbb{Z}_{q}^{n_{1}+n_{2}}$ be a code of cardinality $M<\min \left\{M_{1}, M_{2}\right\}$. Since $M<M_{1}$ there is a set of $s_{1}$ coordinates that disprove $s_{1}$-surjectivity of $C$ with radius $r_{1}$. Form a new code $C^{\prime}$ by projecting $C$ on the set of the other $n_{1}+n_{2}-s_{1}$ coordinates. Since $M<M_{2}$ it holds that $C^{\prime}$ is not $s_{2}$-surjective with radius $r_{2}$, i.e. that $C$ is $\left(s_{1}+s_{2}\right)$-surjective only with radius $\geq\left(r_{1}+1\right)+\left(r_{2}+1\right)>$ $r_{1}+r_{2}+1$. The second inequality follows from (4).

Example: $\sigma_{4}(7,6 ; 3) \geq \min \left\{\sigma_{4}(7,3 ; 1), \sigma_{4}(4,3 ; 1)\right\}=\sigma_{4}(4,3 ; 1)=10$.

The following is a simple yet extremely lower bound, confer e.g. [3, eq. $(2)]$.

Theorem 6. We have $\sigma_{q}(n, s, 0) \geq q \cdot \sigma_{q}(n-1, s-1,0)$.

Proof. Let $C \subset \mathbb{Z}_{q}^{n}$ be an $(s, 0)$-surjective code of cardinality $q \cdot \sigma_{q}(n-1, s-$ $1,0)-1$. Suppose without loss that there are $<\sigma_{q}(n-1, s-1,0)$ words in $C$ with last digit 0 , and let $C^{\prime} \subset \mathbb{Z}_{q}^{n-1}$ be the code obtained by taking all words of $C$ with last digit 0 and then deleting this last digit. If $C$ was $(s, 0)$-surjective, then $C^{\prime}$ would be $(s-1,0)$-surjective, contradicting the fact that $|C|<\sigma_{q}(n-1, s-1,0)$.

The proof of [21, Theorem 4] gives $\sigma_{3}(n, 2 ; 0) \leq 3 \sigma_{2}(n, 2 ; 0)$, another recursive bound.

We finish this section with an unconventional new insight.

Theorem 7. Let $C^{\prime} \subset \mathbb{Z}_{q}^{n}$ be a code of length $n \geq 4$ and

$$
C=\left\{(c_{0}^{\prime}, \ldots, c_{n-1}^{\prime}, \underbrace{c_{n-1}^{\prime}, \ldots, c_{n-1}^{\prime}}_{q \text { times }}) \in \mathbb{Z}_{q}^{n+q} \mid\left(c_{0}^{\prime}, \ldots, c_{n-1}^{\prime}\right) \in C^{\prime}\right\}
$$

another code obtained from the first by repeating the last component $q \geq 2$ times. If $C^{\prime}$ is both, 2-surjective with radius 0 and 4 -surjective with radius 1 , then $C$ is $(q+2)$-surjective with radius $q-1$. 
Proof. Let $k_{1}<\cdots<k_{q+2}$ be coordinates from $\mathbb{Z}_{n+q}$ and $\left(x_{1}, \ldots, x_{q+2}\right) \in$ $\mathbb{Z}_{q}^{q+2}$. We shall show that there is a codeword $c=\left(c_{0}, \ldots, c_{n+q-1}\right) \in C$ such that $x$ is $(q-1)$-covered by $c^{*}$. Set $b=\max \left\{i \in\{1, \ldots, q+2\} \mid k_{i} \in \mathbb{Z}_{n-1}\right\} \geq$ 1. If there are distinct $i, j \in\{b+1, \ldots, q+2\}$ such that $x_{i}=x_{j}$ then there is a $c^{\prime} \in C^{\prime}$ satisfying $c_{k_{1}}^{\prime}=x_{1}$ and $c_{n-1}^{\prime}=x_{i}=x_{j}$ since $C^{\prime}$ is 2-surjective with radius 0 , and the claim follows. Consider now the opposite, which yields $b \geq 2$. If $b=2$ then there is a $c^{\prime} \in C^{\prime}$ satisfying $c_{k_{1}}^{\prime}=x_{1}, c_{k_{2}}^{\prime}=x_{2}$. Furthermore there is a $j \geq 3$ with $c_{n-1}^{\prime}=x_{j}$, since $\left\{x_{i} \in \mathbb{Z}_{q} \mid i \geq 3\right\}=\mathbb{Z}_{q}$, and the claim follows again. If $b=q+2$ or $3 \leq b \leq q+1$ then there is a $c^{\prime} \in C^{\prime}$ satisfying at least three of the four conditions $c_{k_{1}}^{\prime}=x_{1}, c_{k_{2}}^{\prime}=x_{2}$, $c_{k_{3}}^{\prime}=x_{3}$ and $c_{k_{4}}^{\prime}=x_{4}$ or $c_{n-1}^{\prime}=x_{q+2}$, respectively, since $C^{\prime}$ is 4-surjective with radius 1 . This completes the proof.

\section{Exact Results}

Trivially, $\sigma_{1}(n, s ; r)=1=\sigma_{q}(n, s ; s)$ and $K_{q}(n, 0)=q^{n}$.

Theorem 8. If there exists a q-ary $(n, k) M D S$ code then $\sigma_{q}(n, k ; 0)=q^{k}$, otherwise $\sigma_{q}(n, k ; 0)>q^{k}$.

Proof. $\sigma_{q}(n, k ; 0) \geq q^{k}$ is trivial. By definition, an $(n, k)$ MDS code is $k$ surjective with radius 0 . If $C \subset Q^{n}$ is $k$-surjective with radius 0 and $|C|=q^{k}$ then $C$ is an $(n, k)$ MDS code.

The existence problem for MDS codes, i.e. orthogonal arrays of index 1, is discussed for example in [9, Section 2.4].

Corollary 9. $\sigma_{q}(n, n-1 ; 0)=q^{n-1}$.

The next result is a slight generalization of [6, Theorem 3.7.1].

Theorem 10. Let $q \geq 2$ and $t \geq 1$. Then $\sigma_{q}(n, s ; s-t)=q$ if $s \geq(t-1) q+1$ and $\sigma_{q}(n, s ; s-t)>q$ if $s \leq(t-1) q$.

Proof. Analogously to the proof of [6, Theorem 3.7.1] it is easy to see that $\sigma_{q}(n, s ; s-t) \geq q$ and that for $s \geq(t-1) q+1$ the repetition code $\{(a, \ldots, a) \in$ $\left.\mathbb{Z}_{q}^{n} \mid a \in \mathbb{Z}_{q}\right\}$ is $s$-surjective with radius $s-\lceil s / q\rceil \leq s-t$, i.e. $\sigma_{q}(n, s ; s-t)=q$. For $s \leq(t-1) q$ it holds that $\sigma_{q}(n, s ; s-t) \geq K_{q}(s, s-t)>t$ by $(10)$ and [6, Theorem 3.7.1]. 
Non-trivial results are the following.

Theorem 11 (Brace, Daykin [2]; Katona [13] and Rényi [22]; Kleitman, Spencer [18]). $\sigma_{2}(n, 2 ; 0)$ equals the least integer $M$ satisfying

$$
n \leq\left(\begin{array}{c}
M-1 \\
\lfloor M / 2\rfloor-1
\end{array}\right)=\left(\begin{array}{c}
M-1 \\
\lceil M / 2\rceil
\end{array}\right)
$$

Theorem 12 (Johnson, Entringer [11]; Honkala [10]). $\sigma_{2}(n, n-2 ; 0)=$ $\left\lfloor 2^{n} / 3\right\rfloor$.

Sporadic results like $\sigma_{3}(4,3 ; 1)=6$ and $\sigma_{4}(4,3 ; 1)=10$ can be found in $[15,16,7,8]$.

\section{Lower Bounds}

Recall the approach for lower bounds on $\sigma_{q}(n, s ; s-2)$ by partition matrices.

Definition $13([7]) . A q \times n$-matrix $\mathcal{P}=\left(P_{i k}\right)$ of subsets of $\mathbb{Z}_{M}$ is called an $(n, M, q)$-partition matrix if the sets of every column of $\mathcal{P}$ form a partition of $\mathbb{Z}_{M}$. A sequence of $s$ pairwise disjoint subsets from pairwise distinct columns of $\mathcal{P}$ is called an s-transversal.

Theorem $14([7])$. Every $(n, M, q)$-partition matrix has an s-transversal if and only if $\sigma_{q}(n, s ; s-2)>M$.

Theorem 15. $\sigma_{4}(5,3 ; 1) \geq 11$.

Proof. Let $\mathcal{P}$ be a $(5,10,4)$-partition matrix. We shall prove that it contains a 3 -transversal. If $\mathcal{P}$ contains the empty set or every column of $\mathcal{P}$ contains the same 1 -set then the claim follows from $\sigma_{4}(4,2 ; 0)=16$, see [16]. If $\mathcal{P}$ contains a 1-set in one column and a disjoint 1- or 2-set in another column then it is easy to extend them to the desired 3-transversal. Hence, we may assume that every column contains at least two 2 -sets.

Consider now the case that there are sets $Q, \bar{Q}$ in distinct columns of $\mathcal{P}$ satisfying $|Q|=2$ and $Q \subset \bar{Q}$, say $Q=\{0,1\}$ in column 4 and $\bar{Q}$ in column 3 . If on the one hand there is a 2-set $P$ in column $k \in \mathbb{Z}_{3}$ disjoint to $Q$ then we can extend $P, Q$ to a 3 -transversal by using column 3 . If on the other hand every 2 -set of every column of $\mathbb{Z}_{3}$ intersects $Q$ then it is easy to see 
that there are two disjoint 2-sets $\left\{0, a_{0}\right\}$ and $\left\{1, a_{1}\right\}$ in two distinct columns of $\mathbb{Z}_{3}$ which can be extended to a 3-transversal using column 4 .

Consider finally the case that there are no such sets $Q, \bar{Q}$ in $\mathcal{P}$. Call a set big if it contains at least three elements. We will frequently use the following argument: If there is a 2-transversal $\left\{a_{0}, a_{1}\right\},\left\{a_{2}, a_{3}\right\}$ in column $k_{0}, k_{1}$ then we may assume that every set of every column of $\mathbb{Z}_{5} \backslash\left\{k_{0}, k_{1}\right\}$ contains exactly one $a_{i}$ since otherwise it is easy to extend the 2-transversal to a 3 -transversal. Without loss let $\{0,1\}$ and $\{x, y\}$ occur in column 4 . Clearly, there is a column $k \in \mathbb{Z}_{4}$ containing a 2 -set disjoint to $\{0,1\}$ or $\{x, y\}$. Assume without loss that $\{2,3\}$ occurs in column 3. Hence, every set of every column of $\mathbb{Z}_{3}$ contains exactly one element of $\mathbb{Z}_{4}$. Without loss, $\{3,4\}$ occurs in column 2. Every set of every column of $\{0,1,3\}$ contains exactly one element of $\{0,1,3,4\}$. Hence, 2 and 4 are contained together in a big set of column 0 and in a big set of column 1. Without loss, $\{a, 5\}$ occurs in column 2 .

(i) Let $a \in\{0,1\}$, say $a=0$. The 2-transversal $\{2,3\},\{0,5\}$ implies that 1 and 5 are contained together in a big set of column 0 and in a big set of column 1. Thus 0 is in a 2 -set of column 0 and column 1 . The same holds for 3 . Without loss, $\{0,6\}$ and $\{0,7\}$ occur in column 0 and 1 , respectively. Then 1,6,7 are contained together in a big set of column 2 and 3. If column 0 contains $\{3,7\}$ or column 1 contains $\{3,6\}$ then there is a 3 -transversal consisting of this set, $\{0,1\}$ and a set of column 2. Otherwise we may assume that $\{3,8\}$ and $\{3,9\}$ are in column 0 and 1 , respectively. Now column 3 contains $\{4,8,9\}$ and thus also $\{0,5\}$, a contradiction since column 2 also contains $\{0,5\}$.

(ii) Let $a=2$. The 2-transversal $\{0,1\},\{2,5\}$ implies that 3 and 5 are contained together in a big set of column 0 and in a big set of column 1 . Thus 0 is in a 2-set of column 0 and column 1. The same holds for 1 . Without loss, $\{0,6\}$ and $\{0,7\}$ occur in column 0 and 1 , respectively. Then $1,6,7$ are contained together in a big set of column 2 and 3 . If column 0 contains $\{1,7\}$ or column 1 contains $\{1,6\}$ then there is a 3 -transversal consisting of this set, $\{2,3\}$ and a set of column 2 . Otherwise we may assume that $\{1,8\}$ is in column 0 . Now $\{1,8\},\{0,7\}$ can be extended to a 3 -transversal using column 4.

The inequalities (3) and (4) now lead to

$$
K_{4}(8,4) \geq \min \left\{\sigma_{4}(8,3 ; 1), K_{4}(5,2)\right\} \geq \min \left\{\sigma_{4}(5,3 ; 1), 14\right\} \geq 11 .
$$


Recently, Kéri (confer [14]) proved $K_{4}(8,4) \geq 13$ using a computer, here, we got a decent bound by hand.

Research problem: Improve the lower bound of $11 \leq \sigma_{5}(5,4 ; 2) \leq 13$. This would also improve the lower bound of $11 \leq K_{5}(7,4) \leq 21$.

Theorem 16. We have $\sigma_{5}(4,3,1) \geq 14$.

Proof. Kéri proved that the covering code realizing $K_{5}(3,1)$ is unique. The code constructed by Kalbfleisch and Stanton[12] is the union of an MDS-code over $\{0,1\}$ and an MDS-code over $\{2,3,4\}$. Hence, if there was a 3 -surjective code of radius 1 and length 4 over $\mathbb{Z}_{5}$, this code would also be the union of two MDS-codes, however, there does not exist an MDS-code of length 4 over $\mathbb{Z}_{2}$, and our claim follows.

The bound

$$
\sigma_{2}\left(r-1+\left(\begin{array}{l}
\left\lfloor M \cdot 2^{2-r}\right\rfloor-1 \\
\left.\left\lceil M \cdot 2^{2-r}\right\rfloor / 2\right\rceil
\end{array}\right), r ; 0\right)>M
$$

was given in [20, inequality (2)].

\section{Upper Bounds}

Östergård [19] showed that MDS codes can be used to obtain good covering codes. We start with two constructions which are based on the ternary $[4,2]$ Hamming code $H$ with generator matrix

$$
\left[\begin{array}{llll}
0 & 1 & 1 & 1 \\
1 & 0 & 1 & 2
\end{array}\right]
$$

$H$ is well-known to be 1-perfect, especially 1-covering, and $(4,2)$ MDS, especially 2 -surjective with radius 0 . Furthermore, the following statement holds true.

Lemma 17. Let $C \subset \mathbb{Z}_{3}^{n}$ be an $(n, 2) M D S$ code and $k_{1}, k_{2}, k_{3} \in \mathbb{Z}_{n}$ disjoint coordinates. Let $X, Y$ be two 2-subsets of $\mathbb{Z}_{3}$ and $z \in \mathbb{Z}_{3}$. Then there is a $c \in C$ with $c_{k_{1}} \in X$ and $c_{k_{2}} \in Y$ as well as $c_{k_{3}}=z$. 
Proof. For every $x \in X$ there is exactly one $c^{(x)} \in C$ with $c_{k_{1}}^{(x)}=x$ and $c_{k_{3}}^{(x)}=z$. Thus

$$
Y^{\prime}:=\left\{c_{k_{2}}^{(x)} \in \mathbb{Z}_{3} \mid x \in X\right\}
$$

is a 2-set, implying $Y^{\prime} \cap Y \neq \emptyset$.

Set $Q=\mathbb{Z}_{3} \times \mathbb{Z}_{2}$. Let $p_{1}: Q \rightarrow \mathbb{Z}_{3}$ and $p_{2}: Q \rightarrow \mathbb{Z}_{2}$ be projections to the first and second component, respectively.

Theorem 18. $K_{6}(10,7) \leq 18$.

Proof. Consider the ternary linear code $C^{\prime}$ with generator matrix

$$
\left[\begin{array}{llllllllll}
0 & 0 & 0 & 0 & 1 & 1 & 1 & 1 & 1 & 1 \\
1 & 1 & 1 & 1 & 0 & 0 & 1 & 1 & 2 & 2
\end{array}\right]
$$

and set

$$
C:=C^{\prime} \otimes \mathbb{Z}_{2}:=\left\{\left(\left(c_{0}^{\prime}, e\right), \ldots,\left(c_{9}^{\prime}, e\right)\right) \in Q^{10} \mid c^{\prime} \in C^{\prime} \text { and } e \in \mathbb{Z}_{2}\right\} .
$$

We will show that $C \subset Q^{10}$ is a 7-covering code. Let $y \in Q^{10}$. Set $I_{0}=\mathbb{Z}_{4}$, $I_{1}=\{4,5\}, I_{2}=\{6,7\}, I_{3}=\{8,9\}$ as well as $I_{j, e}=\left\{i \in I_{j} \mid p_{2}\left(y_{i}\right)=e\right\} \subset I_{j}$ and $P_{j, e}=\left\{p_{1}\left(y_{i}\right) \in \mathbb{Z}_{3} \mid i \in I_{j, e}\right\} \subset \mathbb{Z}_{3}$. Let $\mathbb{Z}_{4}=\left\{j_{1}, \ldots, j_{4}\right\}$ and choose $e \in \mathbb{Z}_{2}$ such that $\left|I_{0, e}\right|+\left|I_{1, e}\right|+\left|I_{2, e}\right|+\left|I_{3, e}\right| \geq 5$.

(i) If $\left|I_{j_{1}, e}\right|,\left|I_{j_{2}, e}\right|,\left|I_{j_{3}, e}\right|,\left|I_{j_{4}, e}\right| \geq 1$ then $y$ is 7 -covered by $C$ since $H \subset \mathbb{Z}_{3}^{4}$ is 1 -covering.

(ii) If $\left|I_{j_{1}, e}\right|>\left|P_{j_{1}, e}\right|$ then choose distinct $i_{1}, i_{2} \in I_{j_{1}, e}$ with $y_{i_{1}}=y_{i_{2}}$ and choose $i_{3} \in I_{j_{2}, e}$. Since $H$ is 2-surjective with radius 0 , there is a codeword in $C$ which coincides with $y$ in position $i_{1}, i_{2}, i_{3}$. Assume from now that $\left|I_{j_{1}, e}\right|=\left|P_{j_{1}, e}\right|$ and, hence, $\left|I_{j_{1}, e}\right| \leq 3$.

(iii) If $\left|I_{j_{1}, e}\right|=3$ and $\left|I_{j_{2}, e}\right|=\left|I_{j_{3}, e}\right|=1$ then $P_{j_{1}, e}=\mathbb{Z}_{3}$ and $y$ is 7 -covered by $C$ since $H$ is 2 -surjective with radius 0 .

(iv) If $\left|I_{j_{1}, e}\right|,\left|I_{j_{2}, e}\right| \geq 2$ and $\left|I_{j_{3}, e}\right| \geq 1$ then apply Lemma 17 to $H$ to show that $y$ is 7 -covered by $C$.

(v) If $\left|I_{j_{1}, e}\right|=3$ and $\left|I_{j_{2}, e}\right|=2$ and $I_{j_{3}, e}=I_{j_{4}, e}=\emptyset$ then (iv) holds for $I_{j, 1-e}$.

Theorem 19. $K_{6}(9,6) \leq 24$. 
Proof. Consider the ternary linear code $C^{\prime}$ with generator matrix

$$
\left[\begin{array}{lllllllll}
0 & 0 & 0 & 1 & 1 & 1 & 1 & 1 & 1 \\
1 & 1 & 1 & 0 & 0 & 1 & 1 & 2 & 2
\end{array}\right]
$$

Set

$$
C_{0}:=C^{\prime} \otimes\{0\} \text { and } C_{1}:=\left\{\left(\left(c_{8}^{\prime}, 1\right), \ldots,\left(c_{0}^{\prime}, 1\right)\right) \in Q^{9} \mid c^{\prime} \in C^{\prime}\right\}
$$

as well as

$$
\begin{aligned}
C_{2}:=\{( & (\gamma(0), 0),(\gamma(1), 0),(\gamma(2), 0), \bullet, \bullet, \bullet \\
& \left.(\gamma(2), 1),(\gamma(1), 1),(\gamma(0), 1)) \in Q^{9} \mid \gamma \in S_{3}\right\}
\end{aligned}
$$

where each bullet $(\bullet)$ represents an arbitrary element of $Q$ and $S_{3}$ denotes the symmetric group on $\mathbb{Z}_{3}$. We will show that $C_{0} \cup C_{1} \cup C_{2} \subset Q^{9}$ is a 6-covering code. Let $y \in Q^{9}$. Set $I_{0}=\mathbb{Z}_{3}, I_{1}=\{3,4\}, I_{2}=\{5,6\}, I_{3}=\{7,8\}$ as well as $I_{j, 0}=\left\{i \in I_{j} \mid p_{2}\left(y_{i}\right)=0\right\} \subset I_{j}$ and $P_{j, 0}=\left\{p_{1}\left(y_{i}\right) \in \mathbb{Z}_{3} \mid i \in I_{j, 0}\right\} \subset \mathbb{Z}_{3}$. Let $\mathbb{Z}_{4}=\left\{j_{1}, \ldots, j_{4}\right\}$. W.l.o.g. let $\left|I_{0,0}\right|+\left|I_{1,0}\right|+\left|I_{2,0}\right|+\left|I_{3,0}\right| \geq 5$.

If $\left|P_{0,0}\right|=3$ then $y$ is 6 -covered by $C_{2}$. Otherwise $\left|I_{j_{1}, 0}\right|,\left|I_{j_{2}, 0}\right|,\left|I_{j_{3}, 0}\right|,\left|I_{j_{4}, 0}\right| \geq$ 1 or $\left|I_{j_{1}, 0}\right|>\left|P_{j_{1}, 0}\right|$ or $\left|I_{j_{1}, 0}\right|,\left|I_{j_{2}, 0}\right| \geq 2,\left|I_{j_{3}, 0}\right| \geq 1$ and we can argue like in the proof of Theorem 18 to show that $y$ is 6 -covered by $C_{0} \cup C_{1}$.

Soriano has improved this to $K_{6}(9,6) \leq 22$, confer [14].

Theorem 20. If $q \geq 2$ and $s \geq 1$ then $\sigma_{q}(q+s+1, q+s ; q-1) \leq q^{s}$.

Proof. Consider the code $C$ obtained from a $q$-ary $(s+1, s)$ MDS code by repeating the first component $q$ times. We will show that $C$ is $(q+s)$ surjective with radius $q-1$.

(i) Assume $l_{1} \geq q+1$, then there are two distinct $i, j \in \mathbb{Z}_{q+1}$ with $x_{i}=x_{j}$. Thus there is a $c^{*}$ which coincides with $x$ in position $i, j$ and in $s-1$ positions of $\{q+1, \ldots, q+s\} \backslash\left\{l_{1}\right\}$.

(ii) Assume $l_{1} \leq q$. If there are two distinct $i, j \in \mathbb{Z}_{q+1}$ with $x_{i}=x_{j}$ then the situation is analog to (i). Otherwise, $\left\{x_{1}, \ldots, x_{q}\right\}=Q$. Then there is a $c^{*}$ which coincides with $x$ in the $s$ position $q+1, \ldots, q+s$ and in one position of $\mathbb{Z}_{q+1}$.

Theorem 21. If there is a q-ary $(n, 2) M D S$ code $C^{\prime}$ then $\sigma_{q}(q n+n, q n+$ $2 ; q n-n) \leq q^{2}$. 
Proof. Consider the code $C$ obtained from $C^{\prime}$ by repeating every component $(q+1)$-times. We will show that $C$ is $(q n+2)$-surjective with radius $q n-n$. Without loss, we may assume that $l_{1} \geq q+1$. Choose distinct $i_{1}, i_{2} \in$ $\{1, \ldots, q+1\}$ with $x_{i_{1}}=x_{i_{2}}$. Consider the $(n-1,1)$ MDS code $C^{\prime \prime}=$ $\left\{\left(c_{1}^{\prime}, \ldots, c_{n-1}^{\prime}\right) \in Q^{n-1} \mid\left(x_{i_{1}}, c_{1}^{\prime}, \ldots, c_{n-1}^{\prime}\right) \in C^{\prime}\right\}$. No two words of $C^{\prime \prime}$ agree in any position. Hence, there is a $c \in C$ such that $c^{*}$ coincides with $x$ in at least $2+\lceil((q n+2)-(q+1)) / q\rceil=2+n$ positions.

Theorem 22. (i) $\sigma_{2}(7,5 ; 1) \leq 8$.

(ii) $\sigma_{2}(8,6 ; 2), \sigma_{2}(8,4 ; 1) \leq 8$.

(iii) $\sigma_{3}(7,6 ; 3) \leq 6$

(iv) $\sigma_{3}(8,5 ; 2) \leq 9$.

(v) $\sigma_{4}(7,4 ; 2) \leq 8$.

Proof. (i) Consider the dual code $C$ of the binary [7, 4] Hamming code, generated by

$$
G=\left[\begin{array}{lllllll}
1 & 0 & 0 & 1 & 1 & 0 & 1 \\
0 & 1 & 0 & 1 & 0 & 1 & 1 \\
0 & 0 & 1 & 0 & 1 & 1 & 1
\end{array}\right] .
$$

We will show that $C$ is 5 -surjective with radius 1 . Clearly, the columns $l_{1}$ and $l_{2}$ of $G$ contain a row with a zero and a one. Without loss, let this be the upper row. Delete both columns from $G$ to obtain $G^{\prime}$, consisting of the rows $g_{0}, g_{1}, g_{2}$. By construction, $g_{0}$ contains exactly two zeros, say in column $l_{1}^{\prime}$ and $l_{2}^{\prime}$. Thus there is a codeword $c^{\prime} \in \mathbb{Z}_{2}^{5}$ generated by $g_{1}$ and $g_{2}$ such that both $c^{\prime}$ and $c^{\prime}+g_{0}$ coincide with $x$ in position $l_{1}^{\prime}$ and $l_{2}^{\prime}$. Hence, either $c^{\prime}$ or $c^{\prime}+g_{0}$ coincides with $x$ in at least $2+\lceil(5-2) / 2\rceil=4$ positions.

(ii) Consider the binary linear code $C$ generated by

$$
G=\left[\begin{array}{llllllll}
1 & 1 & 0 & 0 & 1 & 1 & 0 & 1 \\
0 & 0 & 1 & 0 & 1 & 0 & 1 & 1 \\
0 & 0 & 0 & 1 & 0 & 1 & 1 & 1
\end{array}\right]
$$

We will first show that $C$ is 6 -surjective with radius 2 . Delete column $l_{1}$ and $l_{2}$ from $G$ to obtain $G^{\prime}$, consisting of $g_{0}, g_{1}, g_{2}$. If $\left\{l_{1}, l_{2}\right\} \notin\{\{2,4\},\{3,5\},\{6,7\}\}$ then $G^{\prime}$ contains a row with exactly three zeros, say in column $l_{1}^{\prime}, l_{2}^{\prime}, l_{3}^{\prime}$. Thus there is a codeword $c^{\prime} \in \mathbb{Z}_{2}^{6}$ generated by $g_{1}$ and $g_{2}$ such that both $c^{\prime}$ and 
$c^{\prime}+g_{0}$ coincide with $x$ in at least two of the three positions $l_{1}^{\prime}, l_{2}^{\prime}, l_{3}^{\prime}$. Hence, $c^{\prime}$ or $c^{\prime}+g_{0}$ coincides with $x$ in at least $2+\lceil(6-3) / 2\rceil=4$ positions. Otherwise $g_{0}$ contains exactly two zeros, say in column $l_{1}^{\prime}, l_{2}^{\prime}$, and there is a codeword $c^{\prime} \in \mathbb{Z}_{2}^{6}$ generated by $g_{1}$ and $g_{2}$ such that both $c^{\prime}$ and $c^{\prime}+g_{0}$ coincide with $x$ in position $l_{1}^{\prime}$ and $l_{2}^{\prime}$. Now the claim follows since $2+\lceil(6-2) / 2\rceil=4$.

We will now show that $C$ is 4 -surjective with radius 1 . Delete the columns with labels in $\mathbb{Z}_{8} \backslash\left\{k_{1}, \ldots, k_{4}\right\}$ from $G$ to obtain $G^{\prime}$, consisting of $g_{0}, g_{1}, g_{2}$. If $k_{2}>1$ then the claim follows from (i), so assume $k_{2}=1$ and let $a \in\{2,3,4\}$ be the number of ones in $g_{0}$. If $a=2$ then the claim follows easily. If $a=3$ then there is a codeword $c^{\prime} \in \mathbb{Z}_{2}^{4}$ generated by $g_{1}$ and $g_{2}$ such that both $c^{\prime}$ and $c^{\prime}+g_{0}$ coincide with $x$ in the unique position containing a zero in $g_{0}$. Hence, either $c^{\prime}$ or $c^{\prime}+g_{0}$ coincides with $x$ in at least $1+\lceil(4-1) / 2\rceil=3$ positions. If $a=4$ then there is a word of $\left\{x_{2} \cdot g_{0}+y_{1} \cdot g_{1}+y_{2} \cdot g_{2} \in \mathbb{Z}_{2}^{4} \mid y_{1}, y_{2} \in \mathbb{Z}_{2}\right\}$ which coincides with $x$ in position 2,3 and 4 .

(iii) Consider the code

$$
\begin{aligned}
C:= & \{(0,0,0,0,0,0,0),(0,0,0,0,0,1,1),(0,0,0,0,0,2,2), \\
& (1,1,1,1,1,0,1),(1,1,1,1,1,1,0),(2,2,2,2,2,2,2)\} \\
= & \left\{c_{1}, c_{2}, c_{3}, c_{4}, c_{5}, c_{6}\right\},
\end{aligned}
$$

say. By symmetry it suffices to consider the case where the first or the last column is deleted, we begin with the latter. If a word $w$ does not contain every letter precisely twice among the first 6 positions, then $w$ contains some letter three times, which means that this word coincides with some codeword in 3 of the first 6 positions. If the 6 th digit is not 0 , then $w$ coincides in the 6th digit with a codeword having only 0 entries at positions 1 to 5 , and we also obtain 3 coincidences. If the 6 th digit is 0 , then $w$ coincides with $c_{4}$ in the 6 th and two of the first 5 positions, and our claim follows.

Now consider the case that the first column is deleted. If the letter 0 or 2 occurs three times, we are immediatelly done. If 1 occurs three times, we are done using $c_{4}$ or $c_{5}$, unless 1 occurs precisely three times, and the last two digits are 1 . Then we are done using $c_{2}$, unless $w$ does not contain 0 , but then 2 has to occur three times, which we already excluded. Hence, every digit occurs precisely twice among the last 6 positions. If none of the last two digits is 0 , then one of $c_{2}, c_{3}$ coincides with $w$ at three positions, if one of them is 0 , then one of $c_{4}, c_{5}$ does. Hence, in any case $w$ coincides with some codeword at 3 positions outside the first column, and our claim follows. 
(iv) Consider the ternary linear code generated by

$$
\left[\begin{array}{llllllll}
0 & 0 & 1 & 1 & 1 & 1 & 1 & 1 \\
1 & 1 & 0 & 0 & 1 & 1 & 2 & 2
\end{array}\right]
$$

Use the ideas of part (i), (ii) and (iv) of the proof of Theorem 18.

(v) Consider

$$
\begin{aligned}
C:= & (0,0,0,3,3,3,3),(0,0,0,0,0,0,0),(1,1,1,0,0,0,2),(1,1,1,1,1,1,1), \\
& (2,2,2,1,1,1,2),(2,2,2,2,2,2,1),(3,3,3,2,2,2,3),(3,3,3,3,3,3,0)\} .
\end{aligned}
$$

We will show that $C$ is 4 -surjective with radius 2 . If $k_{4}<6$ then it is easy to see that $x$ is 2-covered, so assume $k_{4}=6$. Set $A_{0}=A_{3}=\{0,3\}$, $A_{1}=A_{2}=\{1,2\}, B_{0}=\{0,3\}, B_{1}=\{1,2\}, B_{2}=\{0,1\}, B_{3}=\{2,3\}$. Set $I=\left\{i \in\{1,2,3\} \mid k_{i} \in \mathbb{Z}_{3}\right.$ and $x_{i} \in A_{x_{4}}$ or $k_{i} \in \mathbb{Z}_{6} \backslash \mathbb{Z}_{3}$ and $\left.x_{i} \in B_{x_{4}}\right\}$. If $i \in I$ then there is a $c^{*}$ which coincides with $x$ in position $k_{i}$ and 6 . If $I=\emptyset$ then it turns out by inspection that there is a $c^{*}$ which coincides with $x$ in at least two positions of $\left\{k_{1}, k_{2}, k_{3}\right\}$.

Theorem 23. $\sigma_{4}(6,5 ; 2) \leq 48$.

Proof. For $c \in \mathbb{Z}_{4}^{6}$ let $\gamma(c):=\left(c_{1}, c_{0}, c_{3}, c_{2}, c_{5}, c_{4}\right)$. Let

$$
\begin{aligned}
C_{0} & :=\left\{(a, a, b, b, a+b, a+b) \in \mathbb{Z}_{4}^{6} \mid a, b \in \mathbb{Z}_{4}\right\} \\
& =\left\{(a, a, 3 a+b, 3 a+b, b, b) \in \mathbb{Z}_{4}^{6} \mid a, b \in \mathbb{Z}_{4}\right\} \\
& =\left\{(3 a+b, 3 a+b, a, a, b, b) \in \mathbb{Z}_{4}^{6} \mid a, b \in \mathbb{Z}_{4}\right\}
\end{aligned}
$$

and

$$
C_{1}:=\left\{(a, a, b, b, a+3 b, a+3 b) \in \mathbb{Z}_{4}^{6} \mid a, b \in \mathbb{Z}_{4}\right\} .
$$

Let $C_{2}^{\prime}:=\{(0,2,0,2,1,3),(0,2,1,3,0,2),(1,3,0,2,0,2),(1,3,1,3,1,3)\}$ and $C_{2}=\left\{c, \gamma(c) \in \mathbb{Z}_{4}^{6} \mid c \in C_{2}^{\prime}\right\}$. Let $A:=\{(0,1),(2,3)\}$ and $B:=\{(0,3),(1,2)\}$ as well as $C_{3}^{\prime}:=(A \times\{(0,2)\} \times A) \cup(B \times\{(0,2)\} \times B)$ and $C_{3}=\{c, \gamma(c) \in$ $\left.\mathbb{Z}_{4}^{6} \mid c \in C_{3}^{\prime}\right\}$. The code $C=C_{0} \cup C_{1} \cup C_{2} \cup C_{3}$ has cardinality $|C|=$ $4^{2}+4^{2}-2 \cdot 4+2 \cdot 4+2 \cdot 2 \cdot 2^{2}=48$. We will show that $C$ is 5 -surjective with radius 2. Set $y_{k_{j}}=x_{j}$ as well as $y_{l_{1}}=-1$. If $z:=\left|\left\{y_{0}, y_{1}\right\}\right|+\left|\left\{y_{2}, y_{3}\right\}\right|+\left|\left\{y_{4}, y_{5}\right\}\right| \leq 5$ then $x$ is 2 -covered by a $c^{*}$ with $c \in C_{0}$ (and by one with $c \in C_{1}$ ). Assume $z=6$. 
(i) Let $4 \leq l_{1} \leq 5$. If $\left\{y_{0}, y_{1}\right\} \notin\{\{0,2\},\{1,3\}\}$ or $\left\{y_{2}, y_{3}\right\} \notin\{\{0,2\},\{1,3\}\}$ then $\left(\left\{y_{0}, y_{1}\right\}+\left\{y_{2}, y_{3}\right\}\right) \cup\left(\left\{y_{0}, y_{1}\right\}+3 \cdot\left\{y_{2}, y_{3}\right\}\right)=\mathbb{Z}_{4}$ and $x$ is consequently 2 -covered by a $c^{*}$ with $c \in C_{0} \cup C_{1}$. Otherwise $\mid\left(\left\{y_{0}, y_{1}\right\}+\left\{y_{2}, y_{3}\right\}\right) \cup\left(\left\{y_{0}, y_{1}\right\}+\right.$ $\left.3 \cdot\left\{y_{2}, y_{3}\right\}\right) \mid=2$ and it turns out that $x$ is 2 -covered by a $c^{*}$ with $c \in C_{0} \cup C_{1}$ or $c \in C_{2}$.

(ii) Let $0 \leq l_{1} \leq 1$. Analog to (i).

(iii) Let $2 \leq l_{1} \leq 3$. If $\left|\left\{y_{0}, y_{1}, y_{4}, y_{5}\right\}\right|=3$ then $\left(3 \cdot\left\{y_{0}, y_{1}\right\}+\left\{y_{4}, y_{5}\right\}\right) \cup$ $\left(\left\{y_{0}, y_{1}\right\}+3 \cdot\left\{y_{4}, y_{5}\right\}\right)=\mathbb{Z}_{4}$ and $x$ is consequently 2-covered by a $c^{*}$ with $c \in C_{0} \cup C_{1}$. If $\left\{y_{0}, y_{1}\right\},\left\{y_{4}, y_{5}\right\} \in\{\{0,2\},\{1,3\}\}$ then $\mid\left(\left\{y_{0}, y_{1}\right\}+\left\{y_{4}, y_{5}\right\}\right) \cup$ $\left(\left\{y_{0}, y_{1}\right\}+3 \cdot\left\{y_{4}, y_{5}\right\}\right) \mid=2$ and it turns out that $x$ is 2 -covered by a $c^{*}$ with $c \in$ $C_{0} \cup C_{1}$ or $c \in C_{2}$. If $\left\{y_{0}, y_{1}\right\},\left\{y_{4}, y_{5}\right\} \in\{\{0,1\},\{2,3\}\}$ or $\in\{\{0,3\},\{1,2\}\}$ then $\left|\left(\left\{y_{0}, y_{1}\right\}+\left\{y_{4}, y_{5}\right\}\right) \cup\left(\left\{y_{0}, y_{1}\right\}+3 \cdot\left\{y_{4}, y_{5}\right\}\right)\right|=3$ and it turns out that $x$ is 2-covered by a $c^{*}$ with $c \in C_{0} \cup C_{1}$ or $c \in C_{3}$.

The bound [21, Theorem 5] is incorrect for small parameters. An adequate use of the method leads to the implication

$$
\left(\begin{array}{l}
n \\
s
\end{array}\right)<q^{-s}\left(1-q^{-s}\right)^{-M} \Rightarrow \sigma_{q}(n, s ; 0) \leq M,
$$

a weak bound for small parameters, proving e.g. only $\sigma_{2}(6,3 ; 0), \sigma_{3}(5,2 ; 0) \leq$ 39 and $\sigma_{3}(5,3 ; 0) \leq 148$.

\section{Tables}

We restrict our tables for $\sigma_{q}(n, s ; r)$ on $q+n \leq 11$. Furthermore, we only give entries with $q<\sigma_{q}(n, s ; r)<q^{n}$, confer Section 3 .

Key to the tables: Unmarked - [5], Theorem 8, 11 and 12, [14]; a - (4); b - (5); c - [15]; d - [17]; e - (6); f - [7]; g - (8); h - Theorem 5; i - Theorem 15 and 16; $\mathrm{j}$ - (11); k - Theorem 20; $\mathrm{m}$ - Theorem 21; $\mathrm{n}$ - Theorem 22 and 23; o - [8]; p - [16]; q - Theorem 6. 
Table 1. Bounds on $\sigma_{2}(n, s ; r)$

\begin{tabular}{|c|c|c|c|c|}
\hline$n, s \backslash r$ & 0 & 1 & 2 & 3 \\
\hline 3,2 & 4 & & & \\
\hline 4,2 & 5 & & & \\
\hline 4,3 & 8 & & & \\
\hline 4,4 & & 4 & & \\
\hline 5,2 & 6 & & & \\
\hline 5,3 & 10 & & & \\
\hline 5,4 & 16 & $a 4 k$ & & \\
\hline 5,5 & & 7 & & \\
\hline 6,2 & 6 & & & \\
\hline 6,3 & $j 12$ & & & \\
\hline 6,4 & 21 & $a 5 o$ & & \\
\hline 6,5 & 32 & $a 7 o$ & & \\
\hline 6,6 & & 12 & 4 & \\
\hline 7,2 & 6 & & & \\
\hline 7,3 & $j 12$ & & & \\
\hline 7,4 & $j 24$ & o6o & & \\
\hline 7,5 & 42 & $o 8 n$ & & \\
\hline 7,6 & 64 & $a 12-16 g$ & $a 4-7 g$ & \\
\hline 7,7 & & 16 & 7 & \\
\hline 8,2 & 6 & & & \\
\hline 8,3 & $j 12$ & & & \\
\hline 8,4 & $j 24$ & $a 6-8 n$ & & \\
\hline 8,5 & $j 48-56$ & $a 8-24 g$ & & \\
\hline 8,6 & 85 & $a 12-32 g$ & $a 4-8 n$ & \\
\hline 8,7 & 128 & $a 16-32 g$ & $a 7-12 g$ & \\
\hline 8,8 & & 32 & 12 & 4 \\
\hline 9,2 & 6 & & & \\
\hline 9,3 & $j 12$ & & & \\
\hline 9,4 & $j 24$ & $o 7-8 o$ & & \\
\hline 9,5 & $j 48-62$ & $a 8-24 g$ & & \\
\hline 9,6 & $j 96-120$ & $a 12-62 g$ & $a 4-16 g$ & \\
\hline 9,7 & 170 & $a 16-62 g$ & $a 7-16 g$ & \\
\hline 9,8 & 256 & $a 32-62 g$ & $a 12-16 g$ & $a 4 m$ \\
\hline 9,9 & & 62 & 16 & 7 \\
\hline
\end{tabular}


Table 2. Bounds on $\sigma_{3}(n, s ; r)$

\begin{tabular}{|c|c|c|c|c|c|}
\hline$n, s \backslash r$ & 0 & 1 & 2 & 3 & 4 \\
\hline 3,2 & 9 & & & & \\
\hline 3,3 & & 5 & & & \\
\hline 4,2 & 9 & & & & \\
\hline 4,3 & 27 & $c 6 c$ & & & \\
\hline 4,4 & & 9 & & & \\
\hline 5,2 & $p 11$ & & & & \\
\hline 5,3 & $28-33$ & $d 7 d$ & & & \\
\hline 5,4 & 81 & $a 9-27 g$ & & & \\
\hline 5,5 & & 27 & 8 & & \\
\hline 6,2 & $p 12$ & & & & \\
\hline 6,3 & $q 33$ & $d 7 d$ & & & \\
\hline 6,4 & $q 84-111$ & $a 9-33 g$ & & & \\
\hline 6,5 & 243 & $a 27-73 g$ & $a 8-9 k$ & & \\
\hline 6,6 & & $71-73$ & $15-17$ & 6 & \\
\hline 7,2 & $a 12$ & & & & \\
\hline 7,3 & $q 36-40$ & $d 7 d$ & & & \\
\hline 7,4 & $a 84-126$ & $a 9-33 g$ & & & \\
\hline 7,5 & $q 252-377$ & $a 27-111 g$ & $a 8-9 a$ & & \\
\hline 7,6 & 729 & $a 71-186 g$ & $a 15-27 k$ & $a 6 n$ & \\
\hline 7,7 & & $156-186$ & $26-34$ & $11-12$ & \\
\hline 8,2 & $a 12-13$ & & & & \\
\hline 8,3 & $a 36-42$ & $d 7 d$ & & & \\
\hline 8,4 & $a 84-153$ & $a 9-40 g$ & & & \\
\hline 8,5 & $a 252-457$ & $a 27-126 g$ & $a 8-9 n$ & & \\
\hline 8,6 & $q 756-1391$ & $a 71-377 g$ & $a 15-81 g$ & $e 7-9 g$ & \\
\hline 8,7 & 2187 & $a 156-486 g$ & $a 26-81 g$ & $a 10-27 g$ & \\
\hline 8,8 & & $402-486$ & $54-81$ & $14-27$ & 9 \\
\hline
\end{tabular}


Table 3. Bounds on $\sigma_{4}(n, s ; r)$

\begin{tabular}{|c|c|c|c|c|c|}
\hline$n, s \backslash r$ & 0 & 1 & 2 & 3 & 4 \\
\hline 3,2 & 16 & & & & \\
\hline 3,3 & & 8 & & & \\
\hline 4,2 & 16 & & & & \\
\hline 4,3 & 64 & $f 10 f$ & & & \\
\hline 4,4 & & 24 & 7 & & \\
\hline 5,2 & 16 & & & & \\
\hline 5,3 & 64 & $i 11 o$ & & & \\
\hline 5,4 & 256 & $a 24-64 g$ & $c 8 c$ & & \\
\hline 5,5 & & 64 & $15-16$ & & \\
\hline 6,2 & $p 19$ & & & & \\
\hline 6,3 & 64 & $o 12 b$ & & & \\
\hline 6,4 & $257-375$ & $a 24-64 g$ & $a 8 b$ & & \\
\hline 6,5 & 1024 & $a 64-256 g$ & $a 14-48 n$ & & \\
\hline 6,6 & & $228-256$ & $32-52$ & $10-14$ & \\
\hline 7,2 & $a 19-21$ & & & & \\
\hline 7,3 & $q 76-88$ & $a 12 b$ & & & \\
\hline 7,4 & $257-482$ & $a 24-64 g$ & $a 8 n$ & & \\
\hline 7,5 & $q 1028-1764$ & $a 64-375 g$ & $a 14-64 g$ & & \\
\hline 7,6 & 4096 & $a 228-992 g$ & $a 32-128 g$ & $h 10-16 k$ & \\
\hline 7,7 & & $762-992$ & $80-128$ & $16-32$ & $8-10$ \\
\hline
\end{tabular}


Table 4. Bounds on $\sigma_{5}(n, s ; r)$

\begin{tabular}{|c|c|c|c|c|}
\hline$n, s \backslash r$ & 0 & 1 & 2 & 3 \\
\hline 3,2 & 25 & & & \\
\hline 3,3 & & 13 & & \\
\hline 4,2 & 25 & & & \\
\hline 4,3 & 125 & $14 b$ & & \\
\hline 4,4 & & $46-51$ & 11 & \\
\hline 5,2 & 25 & & & \\
\hline 5,3 & 125 & $a 14-17 b$ & & \\
\hline 5,4 & 625 & $a 46-125 g$ & $a 11-13 b$ & \\
\hline 5,5 & & $160-184$ & $21-35$ & 9 \\
\hline 6,2 & 25 & & & \\
\hline 6,3 & 125 & $a 14-18 b$ & & \\
\hline 6,4 & 625 & $a 46-125 g$ & $a 11-13 b$ & \\
\hline 6,5 & 3125 & $a 160-625 g$ & $a 21-125 g$ & $a 9 b$ \\
\hline 6,6 & & & $65-125$ & $13-25$ \\
\hline
\end{tabular}

Table 5. Bounds on $\sigma_{6}(n, s ; r)$

\begin{tabular}{l|cccc}
$n, s \backslash r$ & 0 & 1 & 2 & 3 \\
\hline 3,2 & 36 & & & \\
3,3 & \multicolumn{5}{|c}{18} \\
4,2 & 37 & $a 18 b$ & & \\
4,3 & 216 & 72 & $13-15$ & \\
4,4 & \multicolumn{5}{c}{} \\
\hline 5,2 & $37-39$ & $a 18-22 b$ & & \\
5,3 & $217-240$ & $a 72-216 g$ & $a 13-18 b$ & \\
5,4 & 1296 & $a 72$ & $33-66$ & 12 \\
5,5 & & $330-414$ & $33-$ &
\end{tabular}

Table 6 . Bounds on $\sigma_{7}(n, s ; r)$ and on $\sigma_{8}(n, s ; r)$

\begin{tabular}{|c|c|c|c|c|c|c|c|}
\hline$n, s \backslash r$ & 0 & 1 & 2 & $n, s \backslash r$ & 0 & 1 & 2 \\
\hline 3,2 & 49 & & & 3,2 & 64 & & \\
\hline 3,3 & & 25 & & 3,3 & & 32 & \\
\hline 4,2 & 49 & & & 4,2 & 64 & & \\
\hline 4,3 & 343 & $a 25 b$ & & 4,3 & 512 & $a 32 b$ & \\
\hline 4,4 & & $115-123$ & $17-19$ & 4,4 & & $171-192$ & $22-23$ \\
\hline
\end{tabular}




\section{References}

[1] M.C. Bhandari, C. Durairajan, A note on bounds for $q$-ary covering codes, IEEE Trans. Inform. Theory 42 (1996) 1640-1642.

[2] A. Brace, D.E. Daykin, Sperner type theorems for finite sets, Proc. British Combinatorial Conf. (1972) 18-37.

[3] M. A. Chateauneuf, C. J. Colbourn, D. L. Kreher, Covering arrays of strength three, Des. Codes Cryptogr. 16 (1999), 235-242.

[4] C.J. Colbourn, Covering arrays, in: C.J. Colbourn, J.H. Dinitz (Eds.), Handbook of Combinatorial Designs, 2nd ed., Chapman \& Hall/CRC, Boca Raton, 2007, pp. 361-365.

[5] C.J. Colbourn, Covering Array Tables, http://www.public.asu.edu/ ccolbou/src/tabby/catable.html, accessed February 6, 2009.

[6] G. Cohen, I. Honkala, S. Litsyn, A. Lobstein, Covering Codes, North Holland, Amsterdam, 1997.

[7] W. Haas, J. Quistorff, J.-C. Schlage-Puchta, Lower bounds on covering codes via partition matrices, Journal of Combinatorial Theory, Series A 116 (2009), 478-484.

[8] W. Haas, J. Quistorff, J.-C. Schlage-Puchta, New lower bounds for covering codes, in preparation.

[9] A.S. Hedayat, N.J.A. Sloane, J. Stufken, Orthogonal Arrays, Springer, New York, 1999.

[10] I.S. Honkala, Modified bounds for covering codes, IEEE Trans. Inform. Theory 37 (1991) 351-365.

[11] K.A. Johnson, R. Entringer, Largest induced subgraphs of the $n$-cube that contain no 4-cycles, J. Combin. Theory Ser. B 46 (1989) 346-355.

[12] J. G. Kalbfleisch, R. G. Stanton, A combinatorial problem in matching, J. London Math. Soc. 44 (1969) 60-64. 
[13] G.O.H. Katona, Two applications (for search theory and truth functions) of Sperner type theorems, Periodica Math. Hung. 3 (1973) 19-26.

[14] G. Kéri, Tables for Covering Codes, http://www.sztaki.hu/ keri/codes/, accessed January 22, 2009.

[15] G. Kéri, P.R.J. Östergård, On the covering radius of small codes, Studia Sci. Math. Hungar. 40 (2003) 243-256.

[16] G. Kéri, P.R.J. Östergård, Bounds for covering codes over large alphabets, Des. Codes Cryptogr. 137 (2005) 45-60.

[17] G. Kéri, P.R.J. Östergård, Further results on the covering radius of small codes, Discrete Math. 307 (2007) 69-77.

[18] D.J. Kleitman, J.H. Spencer, Families of $k$-independent sets, Discrete Math. 6 (1973) 255-262.

[19] P.R.J. Östergård, New constructions for $q$-ary covering codes, Ars Comb. 52 (1999) 51-63.

[20] S. Poljak, A. Pultr, V. Rödl, On qualitatively independent partitions and related problems, Discrete Appl. Math. 6 (1983) 193-205.

[21] S. Poljak, Z. Tuza, On the maximum number of qualitatively independent partitions, J. Combin. Theory Ser. A 51 (1989) 111-116.

[22] A. Rényi, Foundations of Probability, Holden-Day, San Francisco, 1970. 